\title{
Socio-demographic determinants of upper gastrointestinal haemorrhage in a teaching hospital

\author{
MA Ahad ${ }^{1}$, MU Ahmed ${ }^{2}$
}

\begin{abstract}
It was a prospective study carried out of all cases of haematemesis and or melaena at Rajshahi Medical College Hospital between December 2004 and March 2005 and determined demographic profile, clinical presentation, cause and outcome of upper gastrointestinal bleeding in a tertiary hospital of Bangladesh. Fifty adult patients presenting with haematemesis and or melaena admitted consecutively into medical units were evaluated through proper history taking, thorough clinical examination, endoscopic examination within 48 hours of first presentation and other related investigations. Patients who were not stabilized haemodynamically within 48 hours of resuscitation and endoscopy could not be done within that period were excluded from this study. Out of 50 patients 44 were male and 6 were female and average age of the patients was 39.9 years. Most of the patients belonged to low socio-economic condition-Farmers $(22 \%)$, Service holder (18\%), Laborers (16\%), Students (14\%), Businessman (8\%), House wife (4\%) and others $(18 \%)$. Farmers, service holders and laborers were the most affected group (57\%). Among the patients monthly income $(\mathrm{Tk})$ was $>20000 /=(2 \%), 15000-20000 /=(8 \%), 8000-15000 /=(10 \%)$ and 0 $8000 /=(80 \%)$. Haematemesis and melaena $(42 \%)$, melaena $(42 \%)$, Haematemesis $(16 \%)$ were the presenting features. Endoscopy revealed that duodenal ulcer (34\%) was the most common cause of upper gastrointestinal (GI) bleeding followed by rupture of portal varices (16\%), neoplasm $(10 \%)$, gastric ulcer $(08 \%)$ and gastric erosion (06\%). Peptic ulcer disease still remains as the major cause of upper (GI) haemorrhage though cases of ruptured oesophageal varices are gradually increasing.
\end{abstract}

Bang Med J (Khulna) 2010; $43: 3-6$

\section{Introduction}

Upper gastrointestinal (GI) bleeding refers to blood loss within the intraluminal gastrointestinal tract from any location between the upper oesophagus to the duodenum at the ligament of Treitz.1 If vomiting occurs shortly after the onset of bleeding, the vomitus appears red and later the appearance is dark red, brown, or black precipitated blood clots in the vomitus and produces a coffee ground appearance. Melaena develops after as little as $50-100 \mathrm{ml}$ of blood loss in upper gastrointestinal tract. Melaena can occur with bleeding of any lesion from areas proximal to and including the caecum. Acute blood loss greater than $50-100 \mathrm{ml}$ may produce melaena for as long as a week. After stool color returns to normal, the test for occult blood test may remain positive for over a week. Stool is black tarry and foul smelling. The black, tarry character of melaena is due to degradation of blood to hematin or other hemochromes by bacteria and should not be confused with greenish character of ingested iron or the black, non foul-smelling stool caused by ingestion of bismuth. Haematemesis results from a combination of large amount of blood filling the stomach together with the urge to vomit. So haematemesis generally indicates a more severe bleeding episode than melaena. Clinical presentation depends on the site, extent and rate of haemorrhage and presence of coincidental disease. Bleeding from the upper GI tract is approximately five times more common than from lower gastrointestinal tract.2,3 It is more common in men and elderly people.2,3 The most common presenting features of upper gastrointestinal haemorrhage are haematemesis, melaena and shock.4 Acute upper gastrointestinal hemorrhage is the most important gastrointestinal emergency and is responsible for about 25,000 admissions to hospital each year in the UK. The incidence is 50$150 / 10000 /$ year and is the highest in areas of social deprivation. It appears that the overall incidence of admissions for upper gastrointestinal bleeding decreased in the UK. In Hong Kong, the incidence has decreased by $30 \%$ over the last 10 years. The mortality of patients admitted to hospital for acute gastrointestinal bleeding is about $10 \%$ in the UK, crude mortality has not changed in more than half a century.5 In Thailand a review of 5000 patients of haematemesis and melaena revealed, peptic ulcer disease $(51 \%)$, acute mucosal erosion $(31.6 \%)$ normal study $(2.46 \%) .6$

In Pakistan, a prospective study of 350 cases who presented with haematemesis, endoscopic study of upper GI tract of them revealed oesophageal varices in $24 \%$ and superficial mucosal lesion in $17 \%$ cases.7 Mortality from bleeding ulcers increased with age. 8 The ASGE9 Survey data suggested that healthy patient less than 60 year old with no underlying disease has a mortality of only $2 \%$. However mortality from a first bleed from varices is around $50 \%$ and most survivors rebleed with inpatient mortality of about $30 \%$. In Bangladesh many patients are admitted in different hospitals with haematemesis and melaena, but there is a little study on the aetiology of the upper gastrointestinal haemorrhage, a survey study by Khan et al (1987) has revealed the point prevalence of

1. Md Abdul Ahad MD, Associate Professor, Dept. of Gastroenterology, Khulna Medical College, Khulna.

2. Mostak Uddin Ahmed MD, Assistant Professor, MAG Osmani Medical College, Sylhet. 
duodenal ulcer (DU) and gastric ulcer (GU) was to be $11.9 \%$ and $3.6 \%$ respectively.10 Alam M T in his study on the aetiology of upper gastrointestinal haemorrhage and its prognosis showed that- duodenal ulcer was the commonest cause of haematemesis and melaena followed by oesophageal varices, gastric ulcer and erosive gastritis. Within 12-24 hours of admission, endoscopy will detect the causes of haemorrhage in about $80 \%$ or more if it is performed expertly.11 Endoscopy should be done within 48 hours of bleeding because if it is delayed, erosions may be healed and ulcer may lose the features like black base or adherent clot which enables the observer to pinpoint them as bleeding sources. In Bangladesh endoscopy is now available in almost every medical college hospitals and many diagnostic centers. So patients with upper GI haemorrhage are evaluated easily.

\section{Materials and Methods}

Fifty adult patients presenting consecutively to Medicine Units in Rajshahi Medical College Hospital with haematemesis and or melaena between December 2004 and March 2005 were included in the present study. Patients presenting with haematemesis and or melaena who were haemodynamically stable were evaluated through proper history taking, thorough clinical examination, endoscopic examination within 48 hours of first presentation and other relevant investigations. Patients, who were not stabilized haemodynamically within 48 hours of resuscitation and endoscopy could not be done within that period were excluded from this study. The clinical history and the examination findings of the patients were noted. History, physical finding and reports of investigations were recorded in a form prepared for this purpose. Patients having clinical features suggestive of upper GI haemorrhage were selected for upper GI endoscopy. The endoscopic examinations were performed by experienced endoscopists using video endoscope. Inclusion Criteria: All the patients clinically suspected of upper GI haemorrhage between 18 years to 90 years. Exclusion Criteria:l. Patients refused endoscopy or did not to give conscent. 2 . Diagnosis of upper GI haemorrhage-not suspected. 3. Failed endoscopy. 4. Comorbid illness 5. Major organ failure. 6. Pregnant women.7. Children under 18 years of age and very elderly patient.

Statistical analysis- Individual patient were given a code number. All data were collected by preformed structured questionnaire from the patient with fulfillment of inclusion and exclusion criteria. At the end of the study the relevant collected data were compiled on a master table first. They were organized by using scientific calculator and standard statistical formula. For the statistical analysis unpaired student's ' $t$ ' test, chi- square test and $Z$ test were performed. PValue $<0.05$ was considered as significant. Appropriate statistical analysis of collected data were done using computer statistical package SPSS 12 version and appropriate statistical method were used to arrive at conclusion making necessary graphs and tables used to write this article.

\section{Results}

Out of 50 patients 44 were male and 6 were female with a male female ratio was 7.33:1. Average age of the patients was 39.9 years with the range of $18-90$ years (Table-I).

Table I

Profile of patients admitted in $\mathrm{RMCH}$

\begin{tabular}{llllllll}
\hline & DU & GU & $\begin{array}{l}\text { Stomal } \\
\text { ulcer }\end{array}$ & $\begin{array}{l}\text { Gastric } \\
\text { erosions }\end{array}$ & $\begin{array}{l}\text { Oeso- } \\
\text { phageal } \\
\text { Varices }\end{array}$ & Tumors & Average \\
\hline $\begin{array}{l}\text { Patients } \\
N=50\end{array}$ & $17(34 \%)$ & $4(8 \%)$ & $1(2 \%)$ & $3(6 \%)$ & $8(16 \%)$ & $5(10 \%)$ & \\
Age & 33.35 & 37.75 & 65 & 34.33 & 49 & 44.2 & 39.9 \\
Male: & & $3: 1$ & $1: 0$ & $3: 0$ & $8: 0$ & $4: 1$ & $7.33: 1$ \\
Female & $16: 1$ & $3: 1$ &
\end{tabular}

Farmers, service holders and laborers were the most affected groups. Laborer cultivators and students suffered more (53\%) from DU. Service holders and businessman suffered more $(75 \%)$ from oesophageal varices. Most (96\%) of the patients were from low socio-economic condition with a mean ( \pm SD) monthly income of taka $4080+2045$ (Table-II).

\section{Table II}

Percentage of occupations and monthly incomes of the patients.

\begin{tabular}{lcc}
\hline 1) Occupations & Numbers & Percentage \\
\hline Farmers & 11 & $22 \%$ \\
Service holder & 9 & $18 \%$ \\
Laborers & 8 & $16 \%$ \\
Students & 7 & $14 \%$ \\
Businessman & 4 & $8 \%$ \\
House wife & 2 & $4 \%$ \\
Others & 9 & $18 \%$ \\
2) Monthly incomes & & \\
Tk.> 20000 & 1 & $2 \%$ \\
Tk. 15000-20000 & 4 & $8 \%$ \\
Tk. 8000-15000 & 5 & $10 \%$ \\
Tk. 0-8000 & 40 & $80 \%$ \\
\hline
\end{tabular}

$68 \%$ patients had blood group ' $\mathrm{O}$ ' and ' $\mathrm{B}$ '. Haematemesis or melaena and both haematemesis and melaena were the presenting feature at $16 \%, 42 \%$ and $42 \%$ of the patients respectively Fig-1. In DU $47.05 \%$ presented with melaena and $41.17 \%$ presented with both haematemesis and melaena. In oesophageal varies $75 \%$ presented with haematemesis and melaena whereas 25\% presented with melaena. "In GU 75\% presented with haematemesis and melaena whereas $25 \%$ presented with melaena. Other presenting features were upper abdominal pain $22 \%$, vomiting $16 \%$ and pain and vomiting $22 \% .42 \%$ of patients presenting with haematemesis and melaena were 
smoker and $8 \%$ gave history of NSAIDs ingestion before occurrence of haematemesis and maelena. Past history of upper abdominal pain was noted in 58.88\% cases. Previous history of jaundice was found in $62.5 \%$ of cases of oesophageal varices. Previous history of haematemesis and melaena was found in $11.76 \%, 25 \%$ and $50 \%$ cases of DU, GU, and oesophageal varices respectively. In general upper abdominal pain was the most $(54 \%)$ common past history.

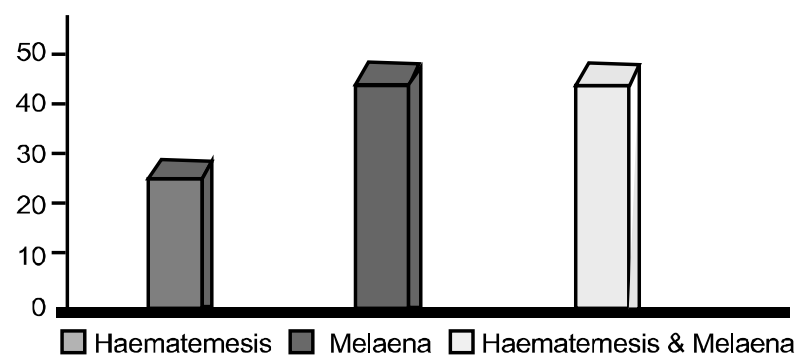

Figure 1 : Proportion of acute upper GI bleeding.

Urgent endoscopic examination within $48-72$ hours of bleeding revealed that duodenal ulcer (34\%) was the most common cause of upper (GI) bleeding followed by rupture of portal varices $(16 \%)$, neoplasm $(10 \%)$, gastric ulcer (8\%) and gastric erosions $(6 \%)$.

\section{Discussion}

Acute upper GI bleeding is a common medical problem that is responsible for significant morbidity and mortality. The extensive clinical spectrum of gastrointestinal bleeding may encompass many different clinical scenarios. It is often very difficult to find out the cause and site of bleeding. Bleeding from the GI tract may present in five ways. Haematemesis is vomitus of red blood or coffee ground material. Melaena is black, tarry, foul smelling stool. Hematochezia is the passage of bright red or maroon blood from rectum. Occult GI bleeding may be identified in the absence of overt bleeding by special examination of the stool (e.g. guaiac testing). Finally, patients may present only with symptoms of blood loss or anemia such as light- headedness, syncope, angina, or dyspneal2. In our study average age of the patients was 39.9 years with the range of 18-90 years. $50 \%$ of patients were in the age range $20-39$ years. This reflects that during the active age of life this disease occurs most. The catchments area of Rajshahi medical college Hospital is populated by those districts where most the people are farmer and labourer. It is also a city of schools, colleges and varsity. Most patients presenting with haematemesis and melaena are cultivators $(22 \%)$, labourers $(16 \%)$ and students $(14 \%)$.This figure may be different in other parts of country. But it should be remembered that poor socioeconomic condition predisposes to $\mathrm{H}$. Pylori infection and poor compliance to anti-ulcer drugs. Monthly income of $80 \%$ of patients was 2000-5000 taka. Lack of money creates difficulty in early diagnosis and treatment of disease and creates complication of peptic ulcer and chronic liver diseases and others. Thus poor and middle class people suffer more from upper (GI) bleeding.

In the current study male female ratio is 11.5:1 for bleeding peptic ulcer. This ratio was 16:1 \& 3:1 for duodenal ulcer and gastric ulcer respectively. This increased incidence of the ratio reflects that male is common sufferers. In the present series, most of the patients with upper gastrointestinal haemorrhage presented with both haematemesis and melaena $42 \%$ cases, melaena in $42 \%$ cases and only haematemesis in $16 \%$ cases. A bleeding duodenal ulcer is likely to be presented with melaena more frequently than haematemesis while a bleeding gastric ulcer patient may present with haematemesis more frequently than melaena. In the present series DU patients presented with haematemesis and melaena in $41.17 \%$ cases, melaena in $47.05 \%$ and haematemesis in $11.76 \%$. In the erosive gastritis $33.33 \%$ presented with haematemesis and $66.66 \%$ presented with both haematemesis and melaena. In variceal group, $75 \%$ of patients presented with both haematemesis and melaena and $25 \%$ with melaena. Bleeding GU presented in $75 \%$ cases with haematemesis and melaena and in 25\% cases with melaena. Variations in presentation in cases of upper GI haemorrhage in different studies may be explained by the fact that haematemesis and melaena is dependent upon the rate, amount and site of bleeding. Peptic ulcer is the commonest cause of haematemesis and melaena.13 In the present study peptic ulcer causing upper GI bleeding in $50 \%$ cases and DU was $34 \%$ of all cases and gastric ulcer was $8 \%$ and stomal ulcer $2 \%$. Variceal bleeding is an important cause of upper GI bleeding In our study $16 \%$ were due to variceal bleeding. The ratio of bleeding duodenal ulcer to gastric ulcer was 4.25:1. Alam14 et al, Mamun et a115. Miah et al16 found the ratio 5.7:1, 11. 66:1, 3.5:1 respectively. Smoking was associated with $35.29 \%$ cases of patients with duodenal ulcer bleeding, 50\% cases of gastric ulcer and $66.66 \%$ cases with erosive gastritis. Oesophagogastro-duodenoscopy is the diagnostic tool in acute upper gastrointestinal haemorrhage17. Duodenal ulcer was diagnosed clinically in 20 patients but at endoscopy it was only 17. Accuracy rate was shown $85 \%$ by Azad khan et al.18 We also found accurate diagnosis in 69.65 of the cases. Many $(72.72 \%)$ cases of clinically suspected variceal bleeding were proved to be correct endoscopicaly. Various literatures showed that in 20$31 \%$ of patients bleeding frequently occured from sources other than varices e.g. from small erosions in the stomach (portal gastropathy). 


\section{Conclusion}

Most of the patients belonged to low socio-economic condition. Farmers, service holders and laborers were the most affected group. Haematemesis and or melaena were the predominant presenting features followed by melaena alone. Endoscopy revealed that duodenal ulcer was the most common cause of upper GI bleeding followed by rupture of portal varices, gastric neoplasm and gastric ulcer. Peptic ulcer disease still remains as the major cause of acute upper gastrointestinal haemorrhage, though cases of ruptured oesophageal varices are gradually increasing.

\section{References}

1. Jutabha R,Jensem DM. Acute Upper Gastrointestinal Bleeding, in.: Friedman SL,McQuaid KR, GrendellJH,Current Diagnosis \& Treatment in Gastroenterology. Mcgraw- Hill 2"d edition inc. 2003; 53.

2. Longstrenth GF. Epidemiology of hospitalization for acute upper gastrointestinal haemorrhage: A population -based study. AMJ Gastroenterol 90: 206, 1995.

3. Longstrenth GF. Epidemiology and outcome of patients hospitalized with acute lower gastrointestinal haemorrhage:A population-based study. AMJ Gastroentero192, 419: 1997. 4. Epstein A, Isselbacher KJ. Gastrointestinal bleeding, In: Harrison's Principle of Internal medicine, 14th Edn. McGraw-Hill Companies Inc.Vol.I. 246-249.

5. Palmer KR. Haematemesis and Melaena, J Med Inter Gastro 2003;17.

6. Roth PH. What is it's role in upper GIT bleeding. Diag Dis Sci 198,26; 7:15-45.

7. Saowaros V,Udayachalem S,Wee Sakal B,Upper gastrointestinal bleeding in Thai patients, review of 5000 upper GIT endoscopy. J Med Assoc Thai 1995; Nov: 77: 561-5.
8. Schiller, K et al. Haemetemesis and melaena with special reference to factors influencing the outcome. Br Med J 1970; 2: 7-14.

9. Chojkier $\mathrm{M}$ et al. Predictors of outcome in massive upper gastrointestinal haemorrhage. $\mathrm{J}$ clin Gastroenterol 1986; 8: 16-22.

10. Khan AKA, Hassan M, Roy PK et al. Peptic ulcer in Bangladesh. Bang Med Res Counc Bull 1987; 111: 29-42.

11. Apel D. Riemann JF, Emergency endoscopy. Can J Gastrcentero12000; Mar; 14: 199-203.

12. Laine Loren; Gastrointestinal bleeding, in Braunwald E, Fauci AS, Dasper DL, Hauser SL, Longo DL, Jameson JL Harrison's Principle of Internal medicine, McGraw-Hill Companies Inc. 2005; 235.

13. Wara P. Incidence, diagnosis and natural course of upper GI haemorrhage: Prognostic value of clinical factors and endoscopy. Scand J Gastroenterol 1978; 22: 26-27.

14. Alam M R. Clinical picture of endoscopically proven peptic ulcer. Bangladesh medical Journal 1982; 10: 137-142.

15. Mamun A A., Clinical evaluation of upper gastrointestinal bleeding, Dissertation 1988-89. 16. Miah, MD. Aetiological pattern of haematemesis and melaena. Dissertation 1996.

17. Chung Y F; Wong WK, Soo KC. Diagnostic failure in endoscopy for acute upper gastrointestinal haemorrhage, Br J Surg 2000; 87: 614-17.

18. Khan AK, Alam MN, Mohsen AQM, Hoque KM. Upper gastrointestinal endoscopy experience in IPGMR, Bang Med J 1982 ;11: 15-22. 